\title{
Very first tests on SOLEIL regarding the Zn environment in pathological calcifications made of apatite determined by X-ray absorption spectroscopy.
}

\author{
D. Bazin ${ }^{1}$, X. Carpentier ${ }^{1}$, O. Traxer $^{2}$, D. Thiaudière ${ }^{3}$, A. Somogyi ${ }^{3}$, S. Reguer ${ }^{3}$, \\ G. Waychunas ${ }^{4}$, M. Daudon ${ }^{5}$ \\ ${ }^{1}$ Laboratoire de Physique des Solides, Bat 510, Université Paris Sud, F91405 Orsay, France. \\ ${ }^{2}$ AP-HP, Hôpital Tenon,Service d’Urologie, 4 Rue de la Chine, 75020 Paris, France \\ ${ }^{3}$ Synchrotron SOLEIL, L'Orme des Merisiers, Saint-Aubin - BP 48, 91192 Gif-sur-Yvette Cedex, France. \\ ${ }^{4}$ Lawrence Berkeley National Laboratory MS 70-108B, 1 Cyclotron Road Berkeley CA 94720, United States. \\ ${ }^{5}$ AP-HP, Hôpital Necker, Service de Biochimie A, 149, Rue de Sèvres, F75743 Paris Cedex 15, France.
}

\begin{abstract}
This very first report of a X-ray absorption spectroscopy experiment on Soleil is part of a more large long term study dedicated to ectopic calcifications. Such biological entities composed of various inorganic and/or organic compounds contain also trace elements. In the case of urinary calculi, different papers already published point out that these oligo elements may promote or inhibit crystal nucleation or growth of mineral or organic species involved. By using such tool specific to synchrotron radiation i.e. determine the local environment of oligoelements and thus their occupation site, we contribute to our understanding of the role of trace elements in ectopic calcifications.
\end{abstract}

\section{Introduction}

Pathological calcifications, associated to very different pathologies ranging from to atherosclerosis to gastric cancer [see for examples 1,2] may be composed of various inorganic and/or organic compounds. If we restrict the scope of such biological entities to kidney stones, the principa component founded for the mineral part, which has a hierarchical structure $[3,4]$ are calcium oxalate $(70 \%$ of the cases), calcium and magnesium phosphates (15\%), uric acid (10\%) and cystine (1\%). By using tools implemented at the hospitals consistent with this chemical complexity such optical microscope and infrared spectrophotometry, it is possible to related their chemical nature and macrostructure to a pathology. For example, in the case of urinary calculi, it has been established a link between cystine and cystinuria while struvite (Magnesium Ammonium Phosphate) kidney stones often called an "infection stone" are usually caused by urinary tract infections with certain types of bacteria. It is also of note that such correlation can be far more subtle, a same chemical phases being related to very different pathologies. In that case, an explanation can be found taking into account the hierarchical structure of the mineral part.

An additional level of complexity is given by the presence of trace elements. Among them, some elements such $\mathrm{Zn}$, due to their special affinity for some crystalline species, may play a role in crystal formation and/or organization of the stones [5]. In previous studies [67], we tried to classify the different elements through synchrotron radiation $\mu$-X-ray fluorescence. Obviously, the presence of major elements which form crystalline phases, i.e. Ca and S ( $\mathrm{S}$ for cystine) are measured, then oligo elements such as Sr, Se, "metal of life" such Fe, Cu and Zn and finally pathological elements such as $\mathrm{Pb}$. Note that the presence of $\mathrm{Sr}$ is linked to the fact that $\mathrm{Ca}$ and $\mathrm{Sr}$ share the same column in the periodic table (same for S and Se). More precisely, the complete set of data which include the different family of kidney stones (whewellite, weddelite, apatite, cystine, uric acid, struvite) is highly suggestive of a substitution process between calcium and the other metals (and not a catalytic one).

We focus the first report of a XAS (X-ray absorption spectroscopy) experiment on Soleil on one of these oligo-elements i.e. on $\mathrm{Zn}$ in order to precise the substitution process. Different studies have already shown that XAS can be employed in order to precise the 
environment of one selected element through its absorption edge. More precisely, information regarding the average coordination geometry as well as the average effective charge are available even for low $\mathrm{Zn}$ concentration materials [8].

\section{Experimental}

\subsection{Material}

The biological samples (Table 1) used in the present study comes from French patients and were first characterized by infrared spectrophotometry using a Fourier transform infrared spectrometer Vector 22 (Bruker Spectrospin, Wissembourg, France) according to the analytical procedure previously described $[9,10]$.

Table 1. Composition as given by Fourier Transform Infra Red Spectroscopy (F.T. I.R.) and the origin of the ectopic calcifications.

\begin{tabular}{|l|l|l|}
\hline Sample & Origin of the calcification & Composition as given by F.T. I.R. \\
\hline N 9009 & kidney & 55\% CA, 38\% MAP, 3\% Prot \\
\hline N 13066 & kidney & 79\% CA, 10\% C2, 7\% Prot \\
\hline N 13086 & Prostatic & 84\% CA, 12\% Prot \\
\hline N 20208 & kidney & 79\% CA, 15\% MAP, ? \\
\hline
\end{tabular}

CA : Carbonate hydroxylapatite , MAP = Magnesium Ammonium Phosphate (struvite)

Prot : Protein, C2 $(\mathrm{Wd})$ = weddellite

Then the stones were investigated either on the H10 beamline [11] at LURE (Orsay, France) or on the New DIFFABS beamline [12] at SOLEIL (St Aubin) in order to determine the electronic state as well as the coordination of Zn atoms. DIFFABS offer the possibility of studying the short and medium-range characteristics of materials by the almost simultaneous combination of XAS and wide angle scattering (WAXS). DIFFABS, situated at the D13-1 bending magnet is equipped with a focused, monochromatic beam ("normal mode", energy range: $3-23 \mathrm{keV}$ ) provided by two bent, Rh-coated, mirrors and a double-crystal Si(111) sagitally focusing monochromator situated in the optic hutch. More details are available on the web site. In our case, the experiment was performed without a focusing device ...

\subsection{Analysis procedure}

X-ray Absorption Near Edge Structure (XANES) spectra were normalized to an edge jump of unity. A prior removal of the background absorption was done by subtraction of a linear function extrapolated from the preedge region.

Following a monoelectronic description of the absorption phenomenon, electronic transitions are allowed between $1 \mathrm{~s}$ and $4 \mathrm{p}$ levels but prohibited toward 3d levels (the dipole selection rule gives $\Delta \mathrm{l}= \pm 1$ ). In fact, hybridizations between $4 \mathrm{p}$ and $3 \mathrm{~d}$ atomic orbitals allow transition between molecular orbitals with $3 \mathrm{~d}$ and $4 \mathrm{p}$ mixed character. At this point, we would like to recall that for an octahedral symmetry, the first unoccupied orbital, t1 $\mathrm{u}^{*}$ possesses a $4 \mathrm{p}$ character and is associated to the main jump observed at 9665-9668 eV for Zn K-edge spectra. The case of tetrahedral symmetry is more complicated [13]. From an experimental point of view, G. A. Waychunas et al. [14] have discussed extensively the form and the position of the Zn edge. In the case of an octahedral first-neighbour anion shell, a strong initial peak followed by a well-defined second peak is measured. Eventually, a small number of other contributions are also visible in the second peak. At the opposite, tetrahedral $\mathrm{Zn}$ 
(willemite, zincite, and franklinite), is associated to a more complex XANES. At least three well-defined edge features can be visible, and there is no large drop in absorption after the first peak, as with the octahedral XANES. In one particular case (franklinite), the edge part of the absorption spectrum is highly unusual and additional detailed structure are measured.

Regarding the XAS oscillations, we can use the Zn-O interatomic distance as a fingerprint of the $\mathrm{Zn}$ geometry. The characteristic $\mathrm{Zn}-\mathrm{O}$ distance in tetrahedral coordination has been reported to be between 1.92 and $1.99 \AA$, while $\mathrm{Zn}$ in octahedral coordination with first-shell $\mathrm{O}$ has a $\mathrm{Zn}-\mathrm{O}$ distance between 2.02 and $2.12 \AA$ [15]. For example, for the following compounds namely $\mathrm{ZnO}, \mathrm{ZnFe}_{2} \mathrm{O}_{4}, \mathrm{Zn}(\mathrm{OH})_{2}, \mathrm{Zn}_{2} \mathrm{SiO}_{4}$, and $\mathrm{CaZn}_{2}(\mathrm{OH})_{6}, 2 \mathrm{H}_{2} \mathrm{O}$ in which $\mathrm{Zn}$ is in tetrahedral coordination, the $\mathrm{Zn}-\mathrm{O}$ distances are respectively equal to 1.98 , $1.98,1.97,1.96$, and $1.94 \AA$.

\section{Results}

The X-ray absorption spectra at the Zn K edge are characterized by two main features, labelled A and B in the figure 1 [16]. The peak A is correlated to transitions over the Fermi level and was found to be sensitive to interatomic distances [17] and/or coordination geometry [18]. The almost constant value of the edge position indicates a relatively constant value for the effective charge of the inserted zinc ions for all compounds.

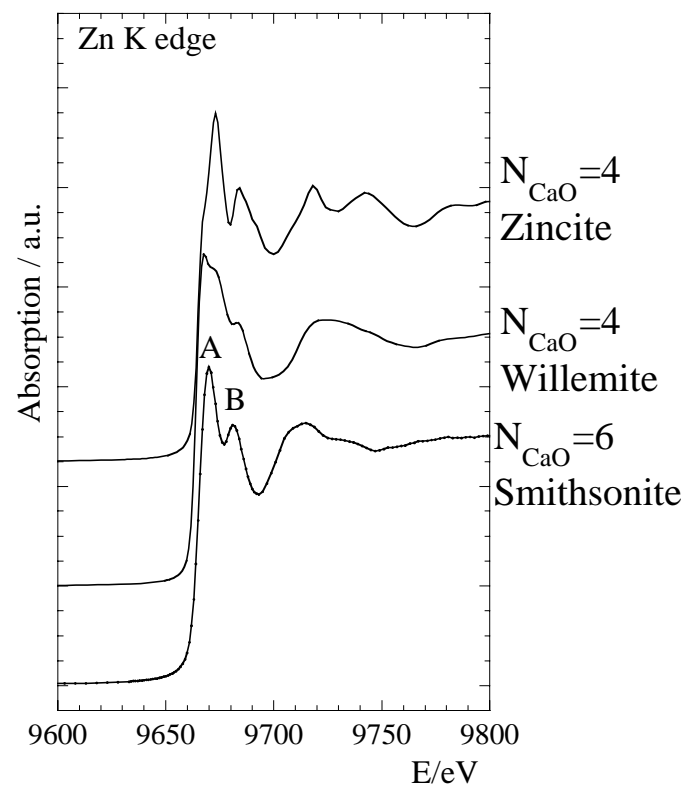

Fig 1

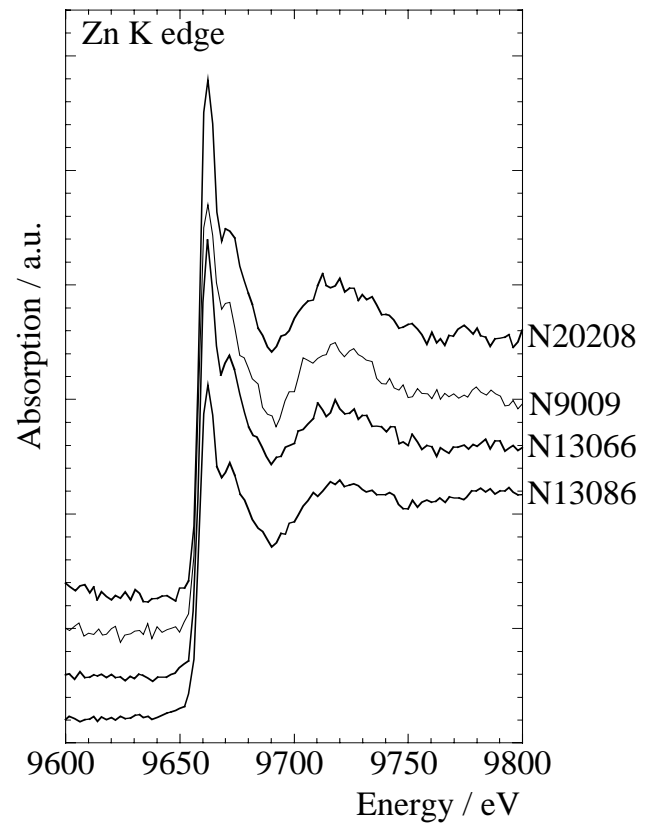

Fig 2

Fig 1 Xanes spectra recorded at the Zn K edge (9659. eV) for a set of references (Zincite, Willemite, Smithsonite). The Zinc environ,ent is indicated in the figure.

Fig 2 Xanes spectra recorded at the Zn K edge (9659. eV) for selected samples

\section{Discussion}

In the case of our biological samples, powder neutron diffraction experiments have been performed. Although the background level is high due to the hydrogen content of this biological sample, the high quality of the diagram allows a complete Rietveld-type refinement using the structural model where hydroxylapatite, $\mathrm{Ca}_{10}\left(\mathrm{PO}_{4}\right)_{6}(\mathrm{OH})_{2}$, has a structure which is 
hexagonal with space group $\mathrm{P}_{3} / \mathrm{m}$ [19;20]. Four $\mathrm{Ca}$ ions, at $\mathrm{Ca}(\mathrm{I})$ sites, lie along columns parallel to the $\mathrm{c}$ axis while six $\mathrm{Ca}$ atoms, positioned at $\mathrm{Ca}$ (II) sites, are laying on the mirror planes. Thus, there are two distinct calcium environments $[21 ; 22], \mathrm{Ca}(\mathrm{I})$ which is best described for the different calcium oxygen bonds as $\mathrm{N}_{\mathrm{CaO}}=6\left(\mathrm{R}_{\mathrm{CaO}}=2.43 \AA\right)+\mathrm{N}_{\mathrm{CaO}}=3$ $\left(\mathrm{R}_{\mathrm{CaO}}=2.79 \AA\right.$ ) coordination environment while $\mathrm{Ca}(\mathrm{II})$ is best described as either $\mathrm{N}_{\mathrm{CaO}}=4$ $\left(\mathrm{R}_{\mathrm{CaO}}=2.36 \AA\right)+\mathrm{N}_{\mathrm{CaO}}=2\left(\mathrm{R}_{\mathrm{CaO}}=2.51 \AA\right)+\mathrm{N}_{\mathrm{CaO}}=1\left(\mathrm{R}_{\mathrm{CaO}}=2.71 \AA\right)$ or $\mathrm{N}_{\mathrm{CaO}}=5\left(\mathrm{R}_{\mathrm{CaO}}=2.35 \AA\right)$ $+\mathrm{N}_{\mathrm{CaO}}=2\left(\mathrm{R}_{\mathrm{CaO}}=2.51 \AA\right)+\mathrm{N}_{\mathrm{CaO}}=1\left(\mathrm{R}_{\mathrm{CaO}}=2.71 \AA\right)$. Note that $\mathrm{Ca}(\mathrm{I})$ and $\mathrm{Ca}(\mathrm{II})$ are present in a $2 / 3$ ratio. Note that in the case of urinary calculi, hydroxylapatite may contain a large amount of carbonate group and thus are denominated as Carbonate apatite (CA).

Here, for all the four selected samples we considered, a careful examination of the Xanes part of the absorption spectra indicates an octahedral complex, Thus, the X-ray absorption spectroscopy indicates that Zinc atoms are probably positionned at $\mathrm{Ca}(\mathrm{I})$ site. At this point, additional developpement of the synchrotron radiation source as well as of the beam line are required to better describe the site occupation of $\mathrm{Zn}$. It is quite clear that a critical evaluation will be made through a careful analysis of the XAS modulations.

\section{Conclusion}

This very first set of experimental tests contributes to our understanding of the role of trace elements in ectopic calcifications. By using tools that are consistent with the chemical complexity of the sample i.e. Powder Neutron Diffraction for the matrix here a biological apatite and XAS for the determination of the coordination number and the electronic state of oligoelements here $\mathrm{Zn}$, the medical community should be better positionned to understand the role of trace element in the formation of the ectopic calcification. Motivated by these observations, a second set of experiment has been scheduled.

\section{Acknowledgment}

G. Waychunas's work was partially supported by the U.S. Department of Energy under Contract No. DE-AC02-05CH11231.

\section{References}

[1] D. F. Lairda, M.R. Mucaloa, and Y. Yokogawa, Growth of calcium hydroxyapatite (Ca-HAp) on cholesterol and cholestanol crystals from a simulated body fluid: A possible insight into the pathological calcifications associated with atherosclerosis, J. of Colloid and Interface Science 295 (2006) 348-363.

[2] L. Balestreri, V. Canzonieri and S. Morassut, Calcified gastric cancer-CT findings before and after chemotherapy case report and discussion of the pathogenesis of this type of calcification Clinical Imaging, 21 (1997) 122-125.

[3] Fratzl P., Characterizing natural fibre composites with hierarchical structure. Fibre Diffraction Rev 2002;210:31-9.

[4] Dujardin E, Mann S., Bio-inspired materials chemistry. Adv Mater 2002;14(11):775-88.

[5] Touryan LA, Lochhead MJ, Marquardt BJ, Vogel V. (2004) Sequential switch of biomineral crystal morphology using trivalent ions, Nature Materials 3: 239-241.

[6] D. Bazin, P. Chevallier, G. Matzen, P. Jungers, M. Daudon, (2003) Heavy elements in urinary stones, Urological Research, 35 179-184.

[7] D. Bazin, M. Daudon, P. Chevallier, S. Rouziere, E. Elkaim, D. Thiaudiere, B. Fayard, E. Foy, P. A. Albouy, G. André, G. Matzen, E. Veron (2006) Les techniques de rayonnement synchrotron au service de la caractérisation d'objets biologiques : un exemple d'application, les calculs rénaux, Annales de Biologie Clinique 64 (2) : 125-39.

[8] D. Bazin, J. Rehr (2003) Soft X-ray absorption spectroscopy at the cutting edge for nanomaterials used in heterogeneous catalysis: The state of the art Catalysis Letters 87(1): 85-90. 
[9] Daudon M, Bader CA, Jungers P (1993) Urinary Calculi: Review of classification methods and correlations with etiology. Scanning microsc 7: 1081

[10] Estepa L, Daudon M (1997) Contribution of Fourier transform infrared Spectroscopy to the identification of urinary stones and kidney crystal deposits. Biospectroscopy. 3:347

[11] Gailhanou, M.; Dubuisson, J. M.; Ribbens, M.; Roussier, L.; Bétaille, D.; Créoff, C.; Lemonnier, M.; Denoyer, J.; Bouillot, C.; Jucha, A.; Lena, A.; Idir, M.; Bessière, M.; Thiaudière, D.; Hennet, L.; Landron, C.; Coutures, J. P. H10 : A materials and high temperature beamline at LURE/DCI. NIM A 2001; 467-468: 745-747.

[12] F. Baudelet, R. Belkhou, V. Briois, A. Coati, P. Dumas, V.H. Etgens, A.M. Flank, P. Fontaine1, Y. Garreau, O. Lyon, I. Quinkal, F. Rochet, P. Roy, M. Sauvage, F. Sirotti, A. Somogyi and D. Thiaudière, SOLEIL a New Powerful Tool for Materials Science, Oil \& Gas Science and Technology - Rev. IFP, Vol. 60 (2005), No. 5, pp. 849-874.

[13] J. Rose, I. Moulin, A.Masion, P. M. Bertsch, M. R. Wiesner, J.-Y. Bottero, F. Mosnier and C. Haehnel (2001) Xas Study of Immobilization Processes for Heavy Metals in Calcium Silicate Hydrates. 2. Zinc, Langmuir 2001, 17, 3658-3665

[14] G. A. Waychunas,C. C. Fuller, J. A. Davis, and J. J. Rehr, Surface complexation and precipitate geometry for aqueous Zn(II) sorption on ferrihydrite:II. XANES analysis and simulation, Geochimica et Cosmochimica Acta, 67 (2003) 1031-1043.

[15] G. Wilkinson, Comprehensive Coordination Chemistry, Pergamon, Oxford, 1987.

[16] M. Giorgetti, S. Passerini,W. H. Smyrl, M. Berrettoni, Identification of an Unconventional Zinc Coordination Site in Anhydrous $\mathrm{ZnxV}_{2} \mathrm{O}_{5}$ Aerogels from Xas, Chem. Mater. 1999, 11, 2257-2264.

[17] A. Bianconi, E. Fritsch, G. Calas and J. Petiau (1985) Xanes of 3d-transition elements in tetrahedral coordination: the effect of bond length variation. Phys. Rev. B, 32, 4292-4295.

[18] Jacquamet L., Aberdam D., Adrait A. Hazemann J.L., Latour J.M., Michaud-Soret I. 1998 X-ray Absorption Spectroscopy of a new zinc site in the FUR Protein from Escherichia Coli. Biochemistry, 37, 2564-2571

[19] M.I. Kay, R.A. Young, A.S. Posner, Crystal structure of hydroxyapatite, Nature, 204, 1050-1052, (1964).

[20] D. Arcos, J. Rodríguez-Carvajal, M. Vallet-Regí, The effect of the silicon incorporation on the hydroxylapatite structure. A neutron diffraction study, Solid State Sciences 6 (2004) 987-994

[21] J. Okasinski, J. B. Cohen, J. Hwang, T. O. Mason, Z. Ding, O. Warschkow and D. E. Ellis, J. Am. Ceram. Soc., 1999, 82, 2451-2459.

[22] F. E. Sowrey, L. J. Skipper, D. M. Pickup, K. O. Drake, Z. Lin, M. E. Smith and R. J. Newport; Systematic empirical analysis of calcium-oxygen coordination environment by calcium K-edge Xanes, Physical Chemistry Chemical Physics 6 (1): 188-192 2004 